\title{
Management Challenges in Atypical Femoral Fractures: A Case Report
}

\author{
Mohammad Golsorkhtabaramiri ${ }^{1}$, Charles A. Inderjeeth ${ }^{2}$ \\ ${ }^{1}$ Department of Rehabilitation and Aged Care, Sir Charles Gairdner Hospital, North Metropolitan Health Service, Perth, Australia \\ ${ }^{2}$ School of Medicine, University of Western Australia, Crawley, Perth, Australia
}

\section{Corresponding Author:}

Mohammad Golsorkhtabaramiri,

Department of Rehabilitation and Aged

Care, Sir Charles Gairdner Hospital,

Nedlands, WA, Australia

E-mail:mgolsorkh@gmail.com

ORCID:

https://orcid.org/0000-0003-3319-4457

Received: November 29, 2019

Revised: February 21, 2020

Accepted: February 21, 2020
A 78-year-old woman presented with an atypical femoral fracture of her right femur associated with a left thigh stress fracture following 9 years of bisphosphonate therapy. The fracture was managed with femoral nailing. However, she represented 3 years later with peri-prosthetic infection, worsening of the left-side stress fracture, and low bone turnover in a bone metabolic study, consistent with an ongoing bisphosphonate effect. This case highlights the increased rate of postoperative complications in atypical femoral fractures even years after surgery as well as the challenging management issues for contralateral fracture and the risk of missing bilateral fractures.

Key Words: Bisphosphonate, Osteoporosis, Femoral fractures

\section{INTRODUCTION}

Bisphosphonates (BPs) have been used for decades to decrease bone fractures in individuals with osteoporosis and those on longterm glucocorticoid therapy. ${ }^{1)}$ A recent study in The New England Journal of Medicine reported good evidence that BPs can decrease the risk of fragility fractures in patients over 65 years of age with osteoporosis and even osteopenia. ${ }^{2)}$ According to the Australian Government's Pharmaceutical Benefits Scheme (PBS), BPs are used for primary prevention in osteopenic patients who have been using $7.5 \mathrm{mg}$ or more of prednisone per day for at least 3 months. As per revised criteria from the American Society for Bone and Mineral Research (ASBMR) task force report, Atypical Femoral Fractures (AFFs) are defined as fractures after no or minimal trauma with non-comminuted ones on femoral diaphysis from distal to the lesser trochanter to proximal to the supracondylar region; with complete ones extended to both cortices and incomplete AFFs involving just lateral cortex (Table 1). ${ }^{1)}$ The risk of AFFs usually increases after 3 years, with a median treatment of 7 years. The relative risk of AFFs is 2.1-128 in patients on BP therapy, with higher risks with long-term use ( $>100$ per 100,000 person-years). ${ }^{1)}$ Management of these fractures and their complications is also challenging for physicians.

We report a case of AFF secondary to long-term BP treatment complicated by a peri-prosthetic wound infection 3 years later in addition to malunion of an incomplete fracture. Compared with other reports, our case highlights the challenging management of asymptomatic contralateral unhealed fractures as well as the risk of delayed postoperative complications even years after surgery in patients with AFFs.

\section{CASE REPORT}

A 78-year-old woman who presented to the hospital with right hip pain after a low-impact fall on her driveway in February 2016 was found to have a transverse fracture of the proximal shaft of her right femur with radiologic findings of AFFs consistent with ASMBR criteria (Table 1, Fig. 1). ${ }^{1)}$ At the same time, a contralateral left thigh radiograph confirmed cortical thickening of the femur, consistent with a stress fracture (Fig. 2). She had previously been treated with risedronate tablets ( $35 \mathrm{mg}$ once weekly) for 9 years from 2006 and had discontinued their use in August 2015. The patient was also on long-term prednisolone (5 $\mathrm{mg}$ daily) to prevent asthma exacerbation. BP treatment was indicated on the basis of long- 
Table 1. ASBMR Task Force 2013 revised criteria for atypical femoral fractures ${ }^{1)}$

\begin{tabular}{ll}
\hline Major criteria & Minimal or no trauma fracture \\
& Transverse fracture line originates from lateral cortex \\
& Complete fracture line involving both cortices associated with medial spike/incomplete fracture on lateral cortex \\
& Non-comminuted or minimally comminuted \\
"Beaking" of fracture site & Generalised increase in diaphyses cortical thickness \\
Minor criteria & Unilateral or bilateral prodromal symptoms \\
& Bilateral complete or incomplete femoral diaphysis fracture \\
& Delayed fracture healing \\
\hline
\end{tabular}

Fracture on femoral diaphysis from distal lesser trochanter to proximal supracondylar ridge.

At least 4 of 5 major criteria need to be present, none of minor criteria necessarily need to be present.

ASBMR; the American Society of Bone and Mineral Research.

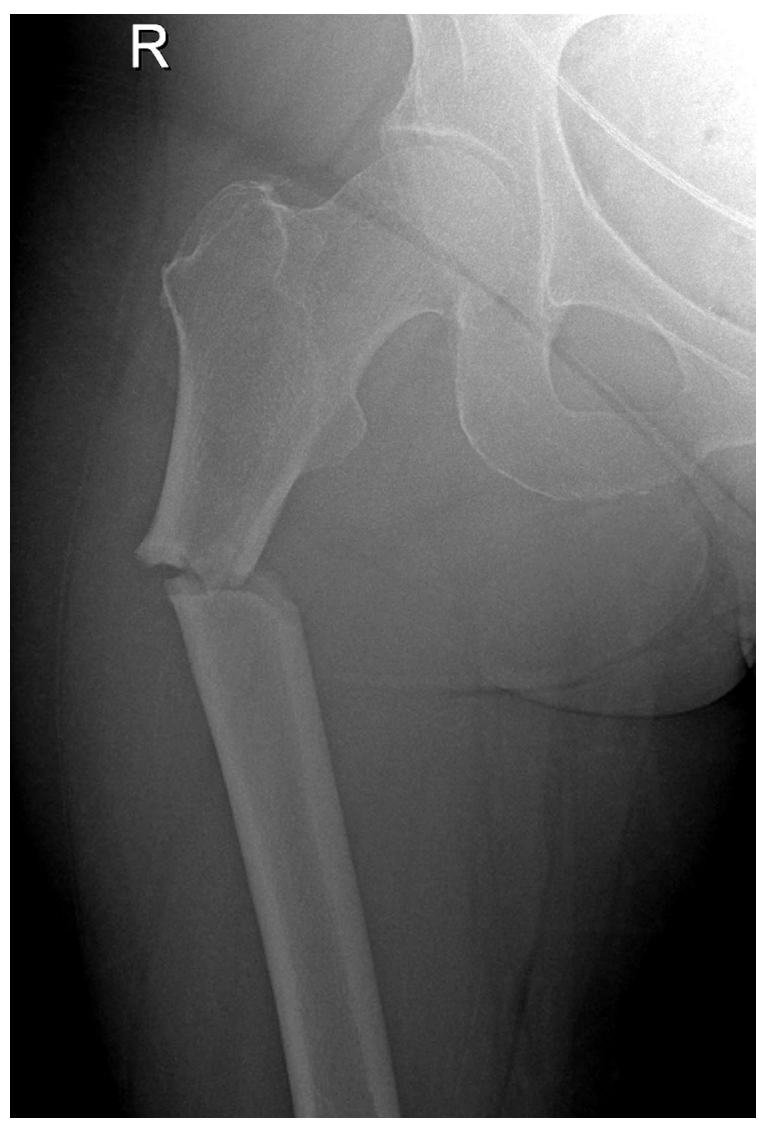

Fig. 1. X-ray of right femur with anteroposterior view showing atypical subtrochanteric fracture in February 2016.

term steroid therapy and osteopenia detected on bone density scans by dual-energy X-ray absorptiometry (DXA). She also had past medical history of asthma, hypertension, and hysterectomy. She underwent antegrade femoral nailing. A survey for secondary causes of her osteoporosis, including evaluation of 25-hydroxyvitamin D, calcium, phosphate, parathyroid hormone (PTH), and thyroid-stimulating hormone levels, serum protein electrophore-

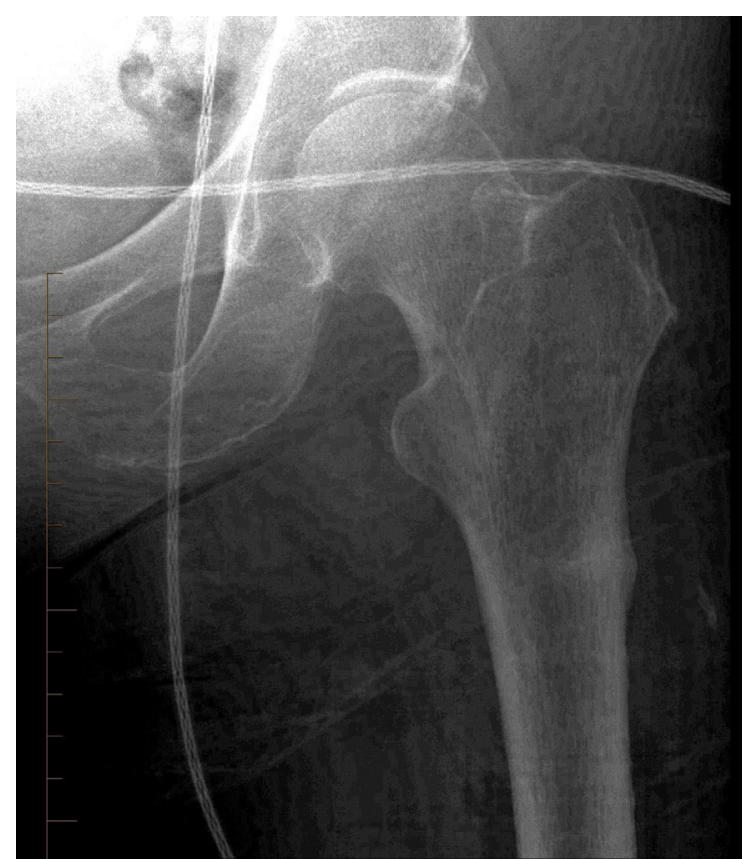

Fig. 2. X-ray of left femur with anteroposterior view showing stress fracture of left femur similar location to right femur in February 2016.

sis, and free light chain assay for multiple myeloma, showed normal results. Despite the incident fracture being considered as AFF, no intervention was considered for the left femur stress fracture because she was asymptomatic at the time. In the hospital, a physiotherapist started her on limited weight-bearing exercises for 6 weeks, which were gradually escalated to full weight-bearing exercises. She was then discharged with calcium and vitamin D supplementation. Her outpatient DXA scan in December 2016 showed an anteroposterior spine $\mathrm{T}$ score of -1.3 , forearm $\mathrm{T}$ score of -0.6 , and total hip $\mathrm{T}$ score of -1.6 , features mainly consistent with those of osteopenia. Comparison with bone densitometry performed in 2015 showed minimal changes. Anabolic therapy with teriparatide 
(TPTD) was considered inappropriate as she did not meet the criteria for the Australian PBS, ${ }^{4)}$ a program for the prescription of subsidized medications offered by the Australian Government.

In January 2019, the patient developed a fever associated with right-sided hip pain that worsened with mobility. A bone scan with technetium 99m-methyl hydroxy diphosphonate ( $\left.{ }^{99 \mathrm{~m}} \mathrm{Tc}-\mathrm{HDP}\right)$ followed by a gallium scan with ${ }^{67} \mathrm{Ga}$ citrate for completion showed peri-prosthetic distal femoral nail infection and ongoing focal activity in the lateral cortex of her left proximal femur, consistent with the stress fracture that remained unhealed after 3 years (Fig. 3). She underwent right femoral nail removal and insertion of a new nail. However, fever, oozing from operation site, and sustained increased inflammatory marker levels after the operation mandated a second operation for removal of the right femoral nail, femur irrigation and debridement, and insertion of an antibiotic-impregnated intramedullary nail (Fig. 4). The patient was transferred to a rehabilitation center with a plan for completion of 6 weeks of intravenous antibiotic therapy in total. She was allowed to weight bear on the right leg and limited weight-bearing on the left leg with a walking frame. Repeated radiography of her left femur confirmed worsening of the stress fracture (Fig. 5).

During this admission, the bone turnover indices were as follows: urine N-terminal telopeptide (NTX), $70 \mathrm{nmol} \mathrm{BCE/L} \mathrm{(ref-}$ erence range, $26-124 \mathrm{nmol} \mathrm{BCE} / \mathrm{mmol}$ creatinine in premenopause); NTX/creatinine ratio, $30 \mathrm{nmol} \mathrm{BCE/L} \mathrm{(>} 50$ in premenopause); ionized serum calcium, $1.28 \mathrm{mmol} / \mathrm{L}(1.12-1.32 \mathrm{mmol} /$ $\mathrm{L})$; plasma phosphate, $1.15 \mathrm{mmol} / \mathrm{L}(0.75-1.50 \mathrm{mmol} / \mathrm{L})$; plasma

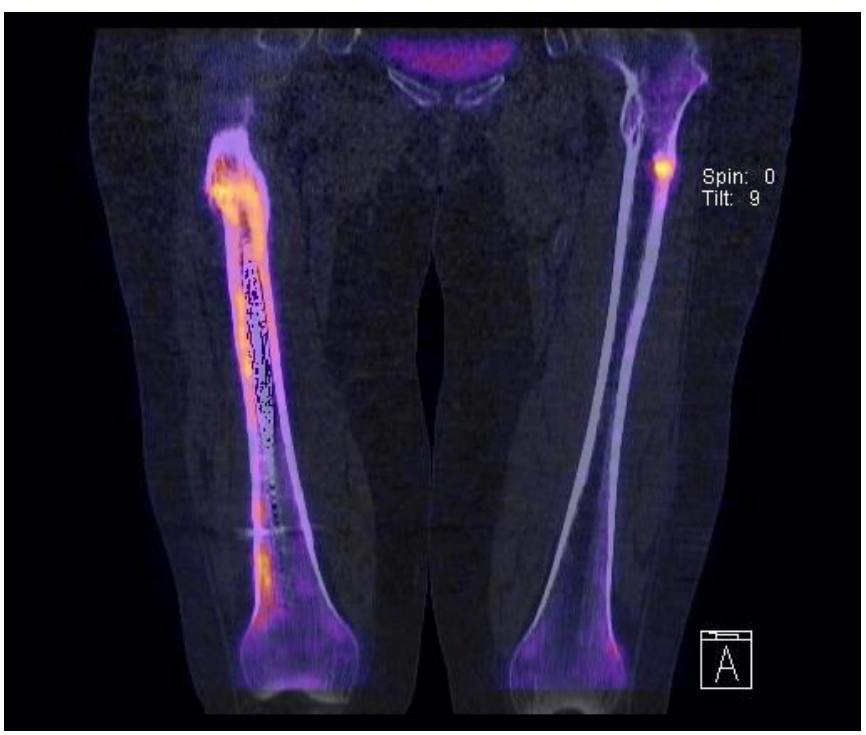

Fig. 3. Bone scan result showing focal activity of left femur consistent with stress fracture in addition to area of hypervascularity in right femur.

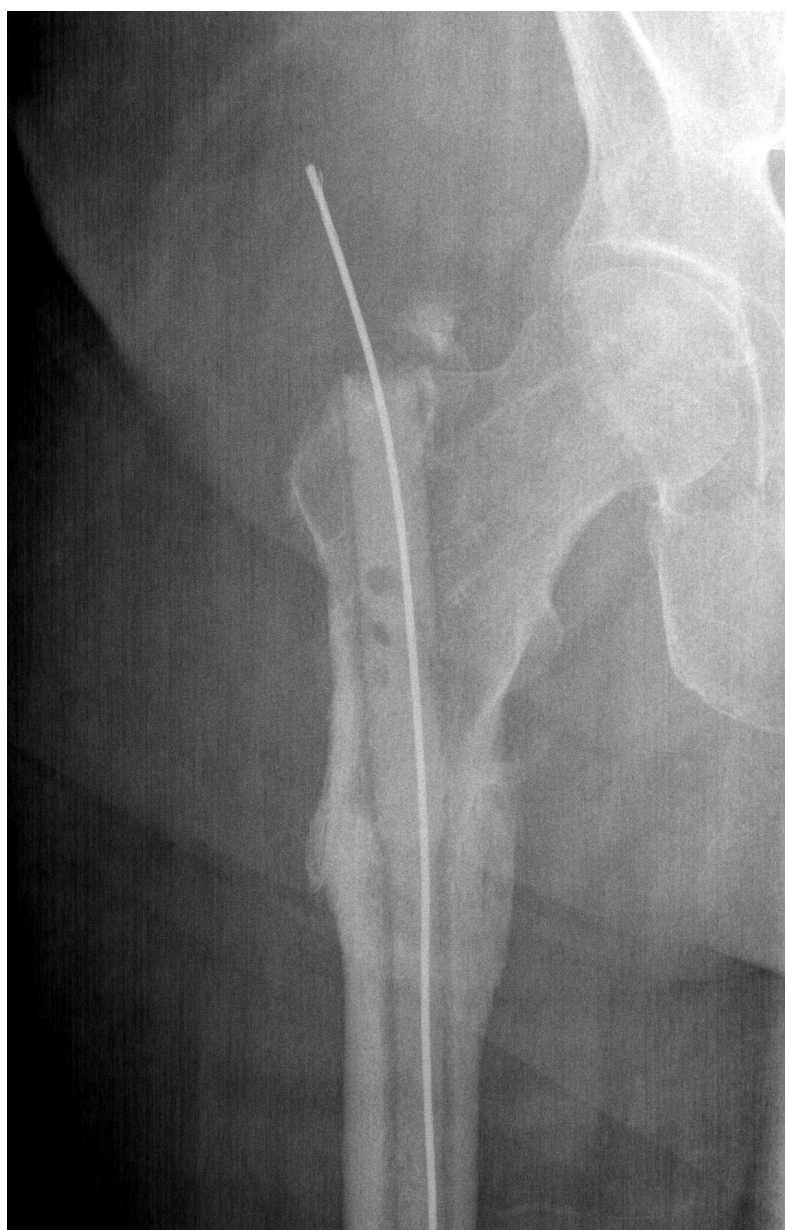

Fig. 4. X-ray of right femur with anteroposterior view showing new intramedullary nail in January 2019.

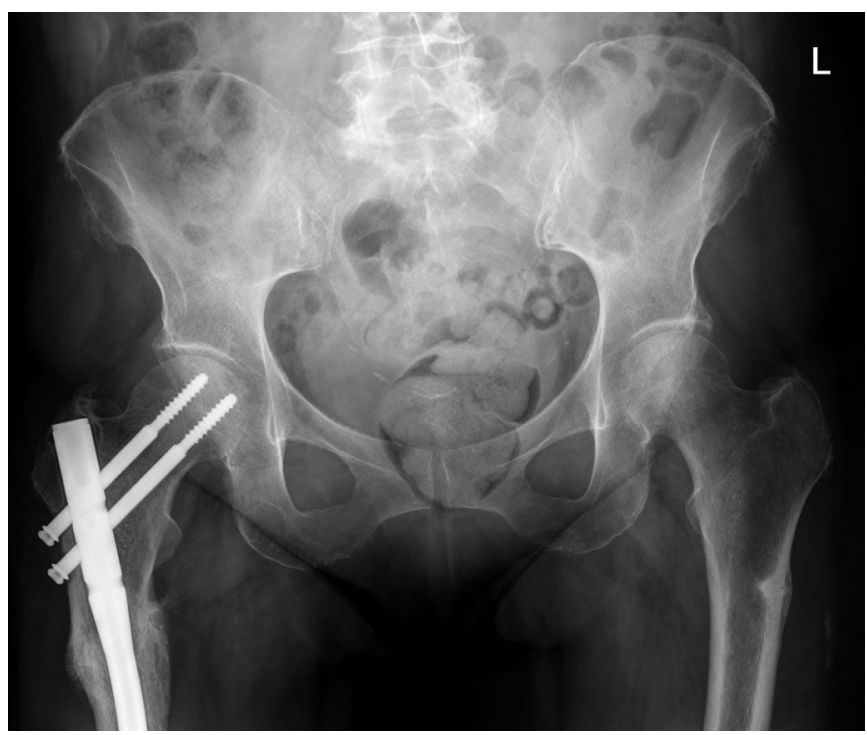

Fig. 5. Pelvis X-ray with anteroposterior view showing right femoral nailing as well as worsening of stress fracture on left femur 3 years later. 
PTH $1.5 \mathrm{pmol} / \mathrm{L}$ (1.6-9.0 pmol/L), bone-specific alkaline phosphatase (BSAP), $0.69 \mathrm{U} / \mathrm{L}$ (30-110 U/L); serum 25-hydroxy vi$\operatorname{tamin} \mathrm{D}, 93 \mathrm{~nm} / \mathrm{L}$ ( > $50 \mathrm{~nm} / \mathrm{L})$, and urine calcium/creatinine ratio, $0.25(0.1-0.58)$. These features were consistent with low bone turnover in the setting of an unhealed fracture. Prophylactic nailing of her left femur stress fracture was performed successfully 2 months after discharge (Fig. 6). The patient returned to baseline function and remained asymptomatic after 8 months of follow-up with callus formation in recovery.

Informed consent obtained from the patient.

\section{DISCUSSION}

The ASMBR uses terms for "stress" and "insufficiency" fractures interchangeably, recognizing stress as augmented pressure on normal bone and insufficiency as excessive pressure on the abnormal bone. ${ }^{1)}$ They are transverse, located on the lateral cortex under the lesser trochanter with a "dreaded black line" appearance in contrast to conventional stress fractures, which are obliquely located on the medial cortex of the proximal femur. BPs can accumulate on stress fracture sites because of increased blood flow and callus formation, which leads to decreased remodeling and increased fracture size. ${ }^{1,5)}$

A systematic literature review of 77 clinical trials including 834 people with complete or incomplete AFFs reported a mean healing time of 7.3 months (2-31 months). A higher failure rate was observed for non-operative treatment of incomplete fractures, whereas prophylactic surgery resulted in a $97 \%$ healing rate. $\left.{ }^{6}\right)$ Moreover, almost $50 \%$ of incomplete fractures can progress to a complete fracture and could eventually require operative management. ${ }^{6)}$ Other studies also demonstrated delayed healing and increased revision rates. ${ }^{7)}$ Indeed, the ASMBR guideline considers delayed healing to be a minor criterion (Table 1). ${ }^{1)}$

Despite conflicting opinions, intramedullary nailing is the favored treatment for complete AFFs. ${ }^{1,6,7)}$ In asymptomatic patients with radiologically incomplete fractures, stress reaction or stress fracture with no pain, the ASMBR guideline suggests limited weight-bearing exercises, calcium and vitamin D supplementation, discontinuation of BP therapy, and monitoring of bone edema until healing occurs. However, due to the high risk of complete fracture, prophylactic nailing is recommended if healing does not occur after 3 months. ${ }^{1)}$

A case series reported an association between intramedullary nailing and with new bone formation in cortical defects in symptomatic incomplete AFFs. ${ }^{8)}$ However, justification of prophylactic surgery in asymptomatic patients can be difficult even in the presence of bilateral disease and previous contralateral fracture. ${ }^{6,7)}$ However, anecdotal studies showed improvement without surgery

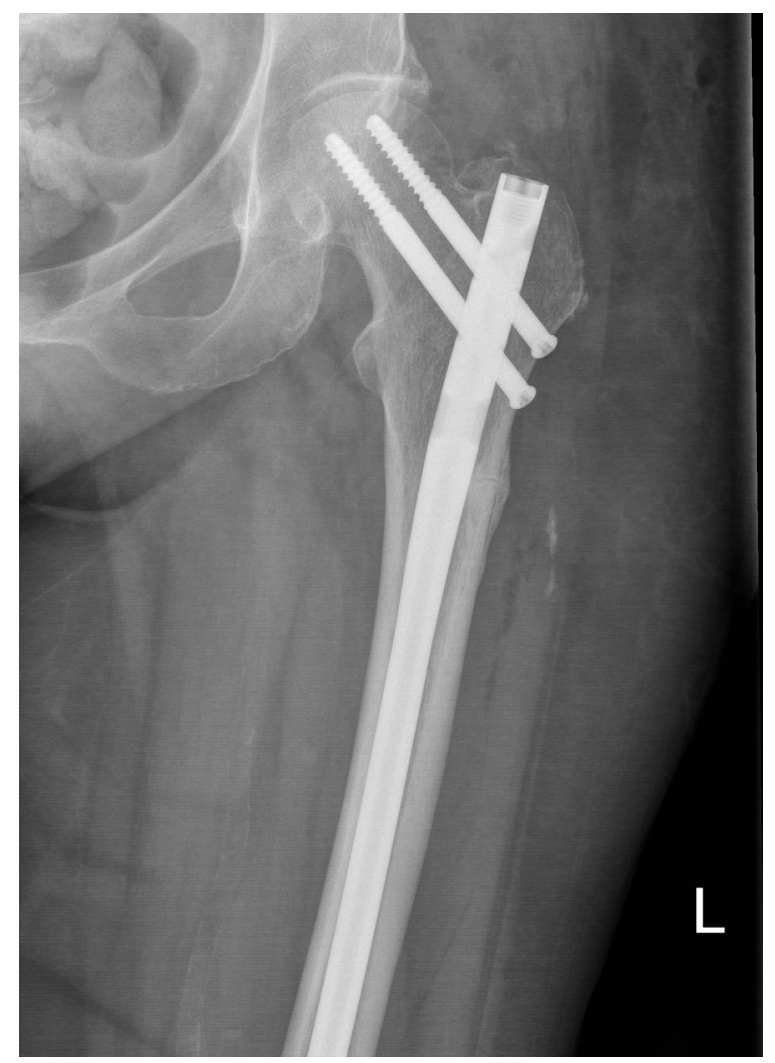

Fig. 6. Anteroposterior view of left femur radiogram after prophylactic nailing in April 2019.

using TPTD as anabolic therapy. ${ }^{9)}$

TPTD, a PTH(1-34) fragment, is another option for the treatment of incomplete AFFs. There is low-quality evidence that TPTD increases bone healing in patients with BP-related AFFs by enhancing bone turnover. ${ }^{7)}$ TPTD can be administered in highrisk patients with $\mathrm{T}$ scores of -3 or less with two or more minimal trauma fractures despite BP treatment for at least 12 months, according to PBS criteria. The treatment period is 18 months ${ }^{4)}$ and is usually followed by antiresorptive treatment to maintain the benefit, which is otherwise lost, which also presents an additional dilemma in patients with AFFs following BP treatment.

Zhang et al. ${ }^{10)}$ reported a case of a 71-year-old woman with osteoporotic vertebral fractures on BPs who developed left-side AFF that was treated with intramedullary nailing. The case was complicated by non-union 2 years later and was conservatively managed with TPTD. However, the patient required revision surgery 5 years post-operation and was also diagnosed with symptomatic incomplete right-side AFF at the same time of developing non-union on the left side. The former was treated with intramedullary nailing and TPTD with excellent bone healing after 3 months. ${ }^{10)}$ Similar to our study, this case also showed that healing can be prolonged in 
asymptomatic AFFs.

In asymptomatic patients, there is always a chance of missing bilateral fracture if there are no symptoms and patients are not screened or monitored for it (radiography, bone scan, CT, or MRI). Another important point is whether to offer prophylactic nailing on the contralateral side, which can be critical for patients because of the risk of contralateral fracture. Good quality randomized clinical trials are needed to assess the benefit of conservative vs. surgical treatment, simply stopping antiresorptive therapy versus anabolic therapy, and other supportive strategies. This remains a dilemma and challenge without a clear answer, and decisions are made on an individual basis according to clinician experience and expertise and patient acceptance of the risks and benefits of each strategy.

\section{ACKNOWLEDGEMENTS}

\section{CONFLICT OF INTEREST DISCLOSURES}

The researchers claim no conflicts of interest.

\section{REFERENCES}

1. Shane E, Burr D, Abrahamsen B, Adler RA, Brown TD, Cheung AM, et al. Atypical subtrochanteric and diaphyseal femoral fractures: second report of a task force of the American Society for Bone and Mineral Research. J Bone Miner Res 2014;29:1-23.

2. Reid IR, Horne AM, Mihov B, Stewart A, Garratt E, Wong S, et al. Fracture prevention with zoledronate in older women with osteopenia. N Engl J Med 2018;379:2407-16.

3. Lu L, Lu L, Zhang J, Li J. Potential risks of rare serious adverse effects related to long-term use of bisphosphonates: an overview of systematic reviews. J Clin Pharm Ther 2020;45:45-51.

4. Australian Department of Health. Schedule of pharmaceutical benefits [Internet]. Sydney: Department of Health; c2019 [cited 2020 Mar 1]. Available from: http://www.pbs.gov.au/medicine/item/9411H.

5. Porrino JA Jr, Kohl CA, Taljanovic M, Rogers LF. Diagnosis of proximal femoral insufficiency fractures in patients receiving bisphosphonate therapy. AJR Am J Roentgenol 2010;194:1061-4.

6. Koh A, Guerado E, Giannoudis PV. Atypical femoral fractures related to bisphosphonate treatment: issues and controversies related to their surgical management. Bone Joint J 2017;99B: 295-302.

7. Larsen MS, Schmal H. The enigma of atypical femoral fractures: a summary of current knowledge. EFORT Open Rev 2018;3: 494-500.

8. Bogl HP, Aspenberg P, Schilcher J. Undisturbed local bone formation capacity in patients with atypical femoral fractures: a case series. Osteoporos Int 2017;28:2439-44.

9. Mailoo VJ, Srinivas V, Turner J, Fraser WD. Beware of bone pain with bisphosphonates. BMJ Case Rep 2019;12:e225385.

10. Zhang HY, Weng HL, Li M, Zhang J. Different surgical outcomes in a patient with bilateral atypical femoral fracture related to bisphosphonate use with or without teriparatide treatment. Osteoporos Int 2019;30:2349-54. 